\title{
Correction to: Isolation and characterization of an antibacterial peptide from protein hydrolysates of Spirulina platensis
}

\author{
Yijun Sun ${ }^{1} \cdot$ Rong Chang ${ }^{2} \cdot$ Qingye $\mathrm{Li}^{2} \cdot$ Bosheng $\mathrm{Li}^{2,3}$
}

Published online: 16 March 2018

c) Springer-Verlag GmbH Germany, part of Springer Nature 2018

Correction to: Eur Food Res Technol (2016) 242:685-692
https://doi.org/10.1007/s00217-015-2576-x

The equal contribution statement was missed to include in the original publication. The correct statement should read as "Yijun Sun and Rong Chang contributed equally to this work".

Bosheng Li

lbosheng0821@163.com

Yijun Sun

sunyijun1213@163.com

1 School of Nature Conservation, Beijing Forestry University, No. 35 Qinghua East Road, Haidian District, Beijing, China

2 College of Biological Sciences and Technology, Beijing Forestry University, No. 35 Qinghua East Road, Haidian District, Beijing, China

3 Beijing Key Laboratory of Forest Food and Safety, Beijing Forestry University, No. 35 Qinghua East Road, Haidian District, Beijing, China 